\title{
Optimal bounds for arithmetic-geometric and Toader means in terms of generalized logarithmic mean
}

Qing Ding ${ }^{1}$ and Tiehong Zhao ${ }^{2^{*}}$

\section{"Correspondence:}

tiehong.zhao@hznu.edu.cn

2Department of Mathematics,

Hangzhou Normal University,

Hangzhou, 311121, China

Full list of author information is

available at the end of the article

\begin{abstract}
In this paper, we find the greatest values $\alpha_{1}, \alpha_{2}$ and the smallest values $\beta_{1}, \beta_{2}$ such that the double inequalities $L_{\alpha_{1}}(a, b)<\operatorname{AG}(a, b)<L_{\beta_{1}}(a, b)$ and

$L_{\alpha_{2}}(a, b)<T(a, b)<L_{\beta_{2}}(a, b)$ hold for all $a, b>0$ with $a \neq b$, where AG $(a, b), T(a, b)$ and $L_{p}(a, b)$ are the arithmetic-geometric, Toader and generalized logarithmic means of two positive numbers $a$ and $b$, respectively.
\end{abstract}

MSC: $26 \mathrm{E} 60$

Keywords: arithmetic-geometric mean; Toader mean; logarithmic mean

\section{Introduction}

For $p \in \mathbb{R}$, the $p$ th generalized logarithmic mean $L_{p}(a, b)$ [1] of two positive numbers $a$ and $b$ is defined by

$$
L_{p}(a, b)= \begin{cases}{\left[\frac{b^{p+1}-a^{p+1}}{(p+1)(b-a)}\right]^{1 / p},} & a \neq b, p \neq-1, p \neq 0, \\ \frac{1}{e}\left(\frac{b^{b}}{a^{a}}\right)^{1 / b-a}, & a \neq b, p=0, \\ \frac{b-a}{\log b-\log a}, & a \neq b, p=-1, \\ a, & a=b .\end{cases}
$$

It is well known that $L_{p}(a, b)$ is continuous and strictly increasing with respect to $p \in \mathbb{R}$ for fixed $a, b>0$ with $a \neq b$. Many remarkable inequalities for the generalized logarithmic mean can be found in the literature [2-17].

The classical arithmetic-geometric mean $\operatorname{AG}(a, b)$ of two positive numbers $a$ and $b$ is defined by starting with $a_{0}=a, b_{0}=b$ and then iterating

$$
a_{n+1}=\frac{a_{n}+b_{n}}{2}, \quad b_{n+1}=\sqrt{a_{n} b_{n}}
$$

for $n \in \mathbb{N}$ until two sequences $\left\{a_{n}\right\}$ and $\left\{b_{n}\right\}$ converge to the same number.

The well-known Gauss identity [18] shows that

$$
\operatorname{AG}(1, r) \mathcal{K}\left(\sqrt{1-r^{2}}\right)=\frac{\pi}{2}
$$

(0) The Author(s) 2017. This article is distributed under the terms of the Creative Commons Attribution 4.0 International License (http://creativecommons.org/licenses/by/4.0/), which permits unrestricted use, distribution, and reproduction in any medium, provided you give appropriate credit to the original author(s) and the source, provide a link to the Creative Commons license, and indicate if changes were made. 
for $r \in(0,1)$, where $\mathcal{K}(r)=\int_{0}^{\pi / 2}\left(1-r^{2} \sin ^{2} t\right)^{-1 / 2} d t, r \in[0,1)$, is the complete elliptic integral of the first kind.

In [19], the Toader mean $T(a, b)$ of two positive numbers $a$ and $b$ was given by

$$
\begin{aligned}
T(a, b)= & \frac{2}{\pi} \int_{0}^{\pi / 2} \sqrt{a^{2} \cos ^{2} \theta+b^{2} \sin ^{2} \theta} d \theta \\
& = \begin{cases}\frac{2 a \mathcal{E}\left(\sqrt{1-(b / a)^{2}}\right)}{\pi}, & a>b, \\
\frac{2 b \mathcal{E}\left(\sqrt{1-(a / b)^{2}}\right)}{\pi}, & a<b, \\
a, & a=b,\end{cases}
\end{aligned}
$$

where $\mathcal{E}(r)=\int_{0}^{\pi / 2}\left(1-r^{2} \sin ^{2} \theta\right)^{1 / 2} d \theta, r \in[0,1]$ is the complete elliptic integral of the second kind.

Recently, the bounds for the arithmetic-geometric mean $\operatorname{AG}(a, b)$ and Toader mean $T(a, b)$ have attracted the attention of many mathematicians. The double inequality

$$
L_{-1}(a, b)=L(a, b)<\operatorname{AG}(a, b)<L^{2 / 3}\left(a^{3 / 2}, b^{3 / 2}\right)
$$

holds for all $a, b>0$ with $a \neq b$. The left inequality of (1.5) was first proposed by Carlson and Vuorinen [20] and also was proved by different methods in [21-23]. Vamanamurthy and Vuorinen [24] proved that $\operatorname{AG}(a, b)<(\pi / 2) L(a, b)$ for all $a, b>0$ with $a \neq b$. The second inequality of (1.5) was proved by Borwein and Borwein [25] and Yang [23].

Vuorinen [26] conjectured that

$$
M_{3 / 2}(a, b)<T(a, b)
$$

for all $a, b>0$ with $a \neq b$, where $M_{p}(a, b)=\left[\left(a^{p}+b^{p}\right) / 2\right]^{1 / p}(p \neq 0)$ and $M_{0}(a, b)=\sqrt{a b}$ is the power mean of order $p$. This conjecture was proved by Qiu and Shen [27] and Barnard et al. [28].

In [29], Alzer and Qiu presented a best possible upper power mean bound for the Toader mean as follows:

$$
T(a, b)<M_{\log 2 / \log (\pi / 2)}(a, b)
$$

for all $a, b>0$ with $a \neq b$.

In [30-32], the authors proved that

$$
\begin{aligned}
& \widehat{L}_{0}(a, b)<T(a, b)<\widehat{L}_{1 / 4}(a, b), \\
& \widehat{S}_{\sqrt{3} / 4}(a, b)<T(a, b)<\widehat{S}_{1 / 2}(a, b)
\end{aligned}
$$

for all $a, b>0$ with $a \neq b$, where $\widehat{L}_{p}(a, b)=\left(a^{p+1}+b^{p+1}\right) /\left(a^{p}+b^{p}\right)$ denotes the $p$ th Lehmer mean and $\widehat{S}_{p}(a, b)$ is the generalized Seiffert mean given by $\widehat{S}_{p}(a, b)=p(a-b) / \arctan [2 p(a-$ $b) /(a+b)](0<p \leq 1, a \neq b), \widehat{S}_{0}(a, b)=(a+b) / 2(a \neq b)$ and $\widehat{S}_{p}(a, a)=a$.

Very recently, Chu and Wang [33] proved that

$$
S_{p_{1}}(a, b)<\operatorname{AG}(a, b)<S_{q_{1}}(a, b),
$$




$$
S_{p_{2}}(a, b)<T(a, b)<S_{q_{2}}(a, b)
$$

for all $a, b>0$ with $a \neq b$ if and only if $p_{1} \leq 1 / 2, q_{1} \geq 1$ and $p_{2} \leq 1, q_{2} \geq 3 / 2$. Here the $p$ th Gini mean of two positive numbers $a$ and $b$ is defined by

$$
S_{p}(a, b)= \begin{cases}\left(\frac{a^{p-1}+b^{p-1}}{a+b}\right)^{1 /(p-2)}, & p \neq 2, \\ \left(a^{a} b^{b}\right)^{1 /(a+b)}, & p=2 .\end{cases}
$$

The main purpose of this paper is to find the greatest values $\alpha_{1}, \alpha_{2}$ and the smallest values $\beta_{1}, \beta_{2}$ such that the double inequalities $L_{\alpha_{1}}(a, b)<\operatorname{AG}(a, b)<L_{\beta_{1}}(a, b)$ and $L_{\alpha_{2}}(a, b)<$ $T(a, b)<L_{\beta_{2}}(a, b)$ hold for all $a, b>0$ with $a \neq b$ and give some new bounds for the complete elliptic integrals.

\section{Basic knowledge and lemmas}

In order to prove our main results, we need several formulas and lemmas, which we present in this section.

For $r \in(0,1)$ and $r^{\prime}=\sqrt{1-r^{2}}$, the well-known complete elliptic integrals of the first and second kinds are defined by

$$
\left\{\begin{array}{l}
\mathcal{K}=\mathcal{K}(r)=\int_{0}^{\pi / 2}\left(1-r^{2} \sin ^{2} \theta\right)^{-1 / 2} d \theta \\
\mathcal{K}^{\prime}=\mathcal{K}^{\prime}(r)=\mathcal{K}\left(r^{\prime}\right) \\
\mathcal{K}(0)=\pi / 2, \quad \mathcal{K}(1)=+\infty
\end{array}\right.
$$

and

$$
\left\{\begin{array}{l}
\mathcal{E}=\mathcal{E}(r)=\int_{0}^{\pi / 2}\left(1-r^{2} \sin ^{2} \theta\right)^{1 / 2} d \theta, \\
\mathcal{E}^{\prime}=\mathcal{E}^{\prime}(r)=\mathcal{E}\left(r^{\prime}\right) \\
\mathcal{E}(0)=\pi / 2, \quad \mathcal{E}(1)=1,
\end{array}\right.
$$

respectively, and the following formulas were presented in [18], Appendix E, pp.474-475:

$$
\begin{aligned}
& \frac{d \mathcal{K}}{d r}=\frac{\mathcal{E}-r^{\prime 2} \mathcal{K}}{r r^{\prime 2}}, \quad \frac{d \mathcal{E}}{d r}=\frac{\mathcal{E}-\mathcal{K}}{r}, \\
& \frac{d\left(\mathcal{E}-r^{\prime 2} \mathcal{K}\right)}{d r}=r \mathcal{K}, \quad \frac{d(\mathcal{K}-\mathcal{E})}{d r}=\frac{r \mathcal{E}}{r^{\prime 2}}, \\
& \mathcal{E}\left(\frac{2 \sqrt{r}}{1+r}\right)=\frac{2 \mathcal{E}-r^{\prime 2} \mathcal{K}}{1+r} .
\end{aligned}
$$

In what follows, four special values $\mathcal{E}(\sqrt{2} / 2), \mathcal{K}(\sqrt{2} / 2)$ and $\mathcal{E}(0.9), \mathcal{K}(0.9)$ will be used. By numerical computations, these are given by

$$
\begin{aligned}
& \mathcal{E}(\sqrt{2} / 2)=1.35064 \cdots, \quad \mathcal{K}(\sqrt{2} / 2)=1.85407 \cdots, \\
& \mathcal{E}(0.9)=1.1717 \cdots, \quad \mathcal{K}(0.9)=2.28055 \cdots .
\end{aligned}
$$


Lemma 2.1 (See [18], Theorem 1.25) For $-\infty<a<b<\infty$, let $f, g:[a, b] \rightarrow \mathbb{R}$ be continuous on $[a, b]$ and be differentiable on $(a, b)$, let $g^{\prime}(x) \neq 0$ on $(a, b)$. If $f^{\prime}(x) / g^{\prime}(x)$ is increasing (decreasing) on $(a, b)$, then so are

$$
\frac{f(x)-f(a)}{g(x)-g(a)} \text { and } \frac{f(x)-f(b)}{g(x)-g(b)}
$$

If $f^{\prime}(x) / g^{\prime}(x)$ is strictly monotone, then the monotonicity in the conclusion is also strict.

Lemma 2.2 (1) The function $r \rightarrow\left(\mathcal{E}-r^{\prime 2} \mathcal{K}\right) / r^{2}$ is strictly increasing from $(0,1)$ onto $(\pi / 4,1)$;

(2) The function $r \rightarrow 2 \mathcal{E}-r^{\prime 2} \mathcal{K}$ is increasing and log-convex from $(0,1)$ onto $(\pi / 2,2)$;

(3) The function $\mathcal{K} / \log \left(e^{2} / r^{\prime}\right)$ is strictly increasing from $(0,1)$ onto $(\pi / 4,1)$;

(4) The function $(\mathcal{K}-\mathcal{E}) / r^{2}$ is strictly increasing on $(0,1)$; in particular, $\mathcal{K}-\mathcal{E}>(\pi / 4) r^{2}$ for all $r \in(0,1)$.

Proof Parts (1) and (2) follow from [18], Theorem 3.21(1) and Exercise 3.43(13).

Lemma 2.3 The equation

$$
(1+p)^{1 / p}=\frac{\pi}{2}
$$

has a unique solution $p=p_{0}=3.15295 \cdots$.

Proof Let

$$
\varphi(p)= \begin{cases}(1+p)^{1 / p}-\pi / 2, & p \in(-1,0) \cup(0,+\infty), \\ e-\pi / 2, & p=0 .\end{cases}
$$

It is easy to verify that the function $\varphi$ is continuous and strictly decreasing from $(-1,+\infty)$ onto $(1,+\infty)$. Therefore, Lemma 2.3 easily follows from the continuity and monotonicity of $\varphi$ together with the facts that $\varphi(3.15295)=6.14999 \times 10^{-7}$ and $\varphi(3.15296)=-4.35155 \times$ $10^{-7}$.

Lemma 2.4 The function

$$
f(r)=\frac{2\left(2 \mathcal{E}-r^{\prime 2} \mathcal{K}\right) / \pi-1-r^{2} / 4}{r^{4}}
$$

is strictly increasing from $(0,1)$ onto $(1 / 64,4 / \pi-5 / 4)$.

Proof Let $f_{1}(r)=2\left(2 \mathcal{E}-r^{\prime 2} \mathcal{K}\right) / \pi-1-r^{2} / 4$ and $\widehat{f}_{1}(r)=r^{4}$, then $f_{1}(0)=\widehat{f}_{1}(0)=0$ and $f(r)=$ $f_{1}(r) / \widehat{f}_{1}(r)$.

A simple calculation yields

$$
\begin{aligned}
& \frac{f_{1}^{\prime}(r)}{\widehat{f}_{1}^{\prime}(r)}=\frac{4\left(\mathcal{E}-r^{\prime 2} \mathcal{K}\right)-\pi r^{2}}{8 \pi r^{4}} \triangleq \frac{f_{2}(r)}{\widehat{f}_{2}(r)}, \\
& f_{2}(0)=\widehat{f}_{2}(0),
\end{aligned}
$$




$$
\begin{aligned}
& \frac{f_{2}^{\prime}(r)}{\widehat{f_{2}^{\prime}}(r)}=\frac{2 \mathcal{K}-\pi}{16 \pi r^{2}} \triangleq \frac{f_{3}(r)}{\widehat{f}_{3}(r)}, \\
& f_{3}(0)=\widehat{f}_{3}(0), \\
& \frac{f_{3}^{\prime}(r)}{\widehat{f}_{3}^{\prime}(r)}=\frac{1}{16 \pi} \cdot \frac{\mathcal{E}-r^{\prime 2} \mathcal{K}}{r^{2}} \cdot \frac{1}{r^{\prime 2}} .
\end{aligned}
$$

Following from Lemma 2.2(1) and (2.9) together with the monotonicity of $1 / r^{\prime 2}$, we clearly see that $f_{3}^{\prime}(r) / \widehat{f}_{3}^{\prime}(r)$ is strictly increasing on $(0,1)$. Equations $(2.5)-(2.8)$ and Lemma 2.1 lead to the conclusion that $f(r)$ is strictly increasing on $(0,1)$.

Therefore, Lemma 2.4 follows from the monotonicity of $f(r)$ together with the facts that $f\left(0^{+}\right)=1 / 64$ and $f\left(1^{-}\right)=4 / \pi-5 / 4$.

The following double inequalities can be obtained from Lemma 2.4 immediately.

\section{Corollary 2.5 Inequalities}

$$
1+\frac{r^{2}}{4}+\frac{r^{4}}{64}<\frac{2}{\pi}\left(2 \mathcal{E}-r^{\prime 2} \mathcal{K}\right)<1+\frac{r^{2}}{4}+\left(\frac{4}{\pi}-\frac{5}{4}\right) r^{4}
$$

hold for $0<r<1$.

Lemma 2.6 The inequality

$$
\left[\frac{(1+r)^{7 / 2}-(1-r)^{7 / 2}}{7 r}\right]^{2 / 5}<1+\frac{r^{2}}{4}
$$

holds for $0<r<1$.

Proof In order to prove inequality (2.10), it suffices to prove that

$$
\begin{aligned}
g(r) & =\left[(1+r)^{7 / 2}-(1-r)^{7 / 2}\right]^{2}-49 r^{2}\left(1+\frac{r^{2}}{4}\right)^{5} \\
& =(1+r)^{7}+(1-r)^{7}-49 r^{2}\left(1+\frac{r^{2}}{4}\right)^{5}-2\left(1-r^{2}\right)^{7 / 2} \\
& =g_{1}(r)-g_{2}(r)<0
\end{aligned}
$$

for $0<r<1$, where

$$
\begin{aligned}
& g_{1}(r)=(1+r)^{7}+(1-r)^{7}-49 r^{2}\left(1+\frac{r^{2}}{4}\right)^{5}, \\
& g_{2}(r)=2\left(1-r^{2}\right)^{7 / 2} .
\end{aligned}
$$

Observe that

$$
\begin{aligned}
& g_{1}^{\prime}(r)=-\frac{7 r}{256}\left[32\left(4-5 r^{2}\right)^{2}+2,848 r^{4}+2,240 r^{6}+350 r^{8}+21 r^{10}\right]<0, \\
& g_{1}(0.56)=0.0755 \cdots>0, \quad g_{1}(0.57)=-0.00966 \cdots<0,
\end{aligned}
$$


we conclude, from (2.12) and (2.13), that there exists $r_{0} \in(0.56,0.57)$ such that $g_{1}(r)>0$ for $r \in\left(0, r_{0}\right)$ and $g_{1}(r)<0$ for $r \in\left(r_{0}, 1\right)$.

In order to prove (2.11), we divide it into two cases.

Case A $r \in\left[r_{0}, 1\right)$. In this case, we clearly see that $g_{1}(r) \leq 0$ and $g_{2}(r)>0$. This implies that $g(r)=g_{1}(r)-g_{2}(r)<0$.

Case B $r \in\left(0, r_{0}\right)$. In this case, $g_{1}(r)>0$. Let $g_{3}(r)=2-7 r^{2}+\frac{35}{4} r^{4}-6 r^{6}$, the difference between $g_{1}(r)$ and $g_{3}(r)$ yields

$$
g_{1}(r)-g_{3}(r)=-\frac{r^{6}\left(10,880+7,840 r^{2}+980 r^{4}+49 r^{6}\right)}{1,024}<0 .
$$

We know from (2.14) that $g_{3}(r)>g_{1}(r)>0$. Moreover,

$$
g_{3}^{2}(r)-g_{2}^{2}(r)=-\frac{r^{6}}{16}\left[\left(2-4 r^{2}\right)\left(4+r^{2}\right)+r^{2}\right]\left[16\left(r^{2}-\frac{5}{8}\right)+\frac{27}{4}\right]<0,
$$

this in conjunction with $g_{3}(r)>0$ implies that

$$
g_{3}(r)-g_{2}(r)<0
$$

Therefore, we clearly see that $g(r)=\left[g_{1}(r)-g_{3}(r)\right]+\left[g_{3}(r)-g_{2}(r)\right]<0$ from $(2.14)$ and (2.15).

Lemma 2.7 Let $\eta(r)=\left[(1+r)^{p_{0}+1}-(1-r)^{p_{0+1}}\right] / r$ and $\omega(r)=\left[(1-r)^{p_{0}}\left(1+p_{0} r\right)-(1+r)^{p_{0}}(1-\right.$ $\left.\left.p_{0} r\right)\right] / r^{2}$, then the functions $\eta(r)$ and $\omega(r)$ both are strictly increasing on $(0,1)$.

Proof We assume that

$$
\begin{aligned}
& \eta_{1}(r)=(1+r)^{p_{0}+1}-(1-r)^{p_{0}+1}, \quad \eta_{2}(r)=r, \\
& \omega_{1}(r)=(1-r)^{p_{0}}\left(1+p_{0} r\right)-(1+r)^{p_{0}}\left(1-p_{0} r\right), \quad \omega_{2}(r)=r^{2},
\end{aligned}
$$

then $\eta(r)=\eta_{1}(r) / \eta_{2}(r)$ and $\omega(r)=\omega_{1}(r) / \omega_{2}(r)$.

A simple calculation yields

$$
\begin{aligned}
& \eta_{1}(0)=\eta_{2}(0)=\omega_{1}(0)=\omega_{2}(0)=0, \\
& \frac{\eta_{1}^{\prime}(r)}{\eta_{2}^{\prime}(r)}=\left(1+p_{0}\right)\left[(1+r)^{p_{0}}-(1-r)^{p_{0}}\right], \\
& \frac{\omega_{1}^{\prime}(r)}{\omega_{2}^{\prime}(r)}=\frac{p_{0}\left(p_{0}+1\right)\left[(1+r)^{p_{0}-1}-(1-r)^{p_{0}-1}\right]}{2} .
\end{aligned}
$$

Lemma 2.1 and (2.16)-(2.18) lead to the conclusion that $\eta(r)$ and $\omega(r)$ are strictly increasing on $(0,1)$.

\section{Lemma 2.8 Let}

$$
\phi_{p}(r)=\frac{2}{\pi}\left(2 \mathcal{E}-r^{\prime 2} \mathcal{K}\right)-\left[\frac{(1+r)^{p+1}-(1-r)^{p+1}}{2(p+1) r}\right]^{1 / p},
$$

then $\phi_{p}(r)>0$ for $0<r<1$ if and only if $p \leq 5 / 2 ; \phi_{p}(r)<0$ for $0<r<1$ if and only if $p \geq p_{0}$. 
Proof It is well known that $L_{p}(a, b)$ is strictly increasing with respect to $p \in \mathbb{R}$ for fixed $a, b>0$ with $a \neq b$, then $\phi_{p}(r)$ is strictly decreasing with respect to $p \in \mathbb{R}$. In order to prove Lemma 2.8, we divide it into three cases.

Case $1 p=5 / 2$.

From Corollary 2.5 and Lemma 2.6, we clearly see that

$$
\begin{aligned}
\phi_{5 / 2}(r) & =\frac{2}{\pi}\left(2 \mathcal{E}-r^{\prime 2} \mathcal{K}\right)-\left[\frac{(1+r)^{7 / 2}-(1-r)^{7 / 2}}{7 r}\right]^{2 / 5} \\
& >1+\frac{r^{2}}{4}+\frac{r^{4}}{64}-\left[\frac{(1+r)^{7 / 2}-(1-r)^{7 / 2}}{7 r}\right]^{2 / 5} \\
& >\frac{r^{4}}{64}>0
\end{aligned}
$$

for $0<r<1$.

Case $2 p=p_{0}$.

We divide it into two subcases.

Subcase A $\phi_{p_{0}}(r)<0$ for $r \in(0,0.9)$.

Since $\phi_{p}(r)$ is strictly decreasing with respect to $p \in \mathbb{R}$, we clearly see that $\phi_{p_{0}}(r)<\phi_{3}(r)$. It suffices to prove that $\phi_{3}(r)<0$ for $r \in(0,0.9)$.

For $r \in(0, \sqrt{2} / 2]$, it follows from Corollary 2.5 that

$$
\begin{aligned}
\phi_{3}(r) & =\frac{2}{\pi}\left(2 \mathcal{E}-r^{\prime 2} \mathcal{K}\right)-\left[\frac{(1+r)^{4}-(1-r)^{4}}{8 r}\right]^{1 / 3} \\
& <1+\frac{r^{2}}{4}+\left(\frac{4}{\pi}-\frac{5}{4}\right) r^{4}-\left(1+\frac{r^{2}}{3}-\frac{r^{4}}{9}\right)^{3} \\
& =-\frac{r^{2}}{12}\left[1-\left(\frac{48}{\pi}-\frac{41}{3}\right) r^{2}\right] \\
& \leq-\frac{r^{2}}{12}\left[1-\frac{1}{2}\left(\frac{48}{\pi}-\frac{41}{3}\right)\right] \\
& =-\frac{(47 \pi-144) r^{2}}{72 \pi}<0,
\end{aligned}
$$

where the first inequality easily follows from

$$
\frac{(1+r)^{4}-(1-r)^{4}}{8 r}-\left(1+\frac{r^{2}}{3}-\frac{r^{4}}{9}\right)^{3}=\frac{r^{6}}{729}\left[126+9\left(1-r^{4}\right)+r^{6}\right]>0
$$

For $r \in(\sqrt{2} / 2,0.9)$, taking the derivative of $\phi_{3}(r)$ yields

$$
\phi_{3}^{\prime}(r)=\frac{2\left(\mathcal{E}-r^{\prime 2} \mathcal{K}\right)}{\pi r}-\frac{2 r}{3\left(1+r^{2}\right)^{2 / 3}}=\mu_{1}(r)+\mu_{2}(r)
$$

where

$$
\mu_{1}(r)=\frac{2\left(\mathcal{E}-r^{\prime 2} \mathcal{K}\right)}{\pi r}-\frac{r}{2}, \quad \mu_{2}(r)=\frac{r}{2}-\frac{2 r}{3\left(1+r^{2}\right)^{2 / 3}}
$$


From Lemma 2.2(4), we clearly see that

$$
\frac{d \mu_{1}(r)}{d r}=\frac{2}{\pi r^{2}}\left(\mathcal{K}-\mathcal{E}-\frac{\pi}{4} r^{2}\right)>0
$$

for $r \in(0,1)$ and

$$
\begin{aligned}
\frac{d \mu_{2}(r)}{d r} & =\frac{9\left(1+r^{2}\right)^{5 / 3}-12+4 r^{2}}{18\left(1+r^{2}\right)^{5 / 3}}>\frac{9\left[1+(5 / 3) r^{2}\right]-12+4 r^{2}}{18\left(1+r^{2}\right)^{5 / 3}} \\
& =\frac{19 r^{2}-3}{18\left(1+r^{2}\right)^{5 / 3}}>0
\end{aligned}
$$

for $r \in(\sqrt{2} / 2,0.9)$. Equations (2.19)-(2.21) lead to the conclusion that $\phi_{3}^{\prime}(r)$ is strictly increasing on $(\sqrt{2} / 2,0.9)$. This in conjunction with $(2.3)$ implies that

$$
\phi_{3}^{\prime}(r)>\phi_{3}^{\prime}(\sqrt{2} / 2)=0.02163 \cdots>0
$$

for $r \in(\sqrt{2} / 2,9 / 10)$. Therefore, from (2.22) we clearly see that $\phi_{3}(r)$ is strictly increasing on $(\sqrt{2} / 2,0.9)$. This in conjunction with $(2.4)$ yields $\phi_{3}(r)<\phi_{3}(0.9)=-0.002687 \cdots<0$ for $r \in(\sqrt{2} / 2,0.9)$.

Subcase B $\phi_{p_{0}}(r)<0$ for $r \in[0.9,1)$.

For $0.9 \leq r<1$, taking the derivation of $\phi_{p_{0}}(r)$ yields

$$
\phi_{p_{0}}^{\prime}(r)=\frac{2\left(\mathcal{E}-r^{\prime 2} \mathcal{K}\right)}{\pi r}-\frac{\omega(r)}{p_{0}\left(p_{0}+1\right) 2^{1 / p_{0}} \eta(r)^{1-1 / p_{0}}},
$$

where $\omega(r)$ and $\eta(r)$ are defined as in Lemma 2.7. From Lemma 2.2(1), we clearly see that $\left(\mathcal{E}-r^{\prime 2} \mathcal{K}\right) / r$ is strictly increasing on $(0,1)$. Lemma 2.7 and $(2.4),(2.20)$ lead to the conclusion that

$$
\begin{aligned}
\phi_{p_{0}}^{\prime}(r) & \geq \frac{2\left[\mathcal{E}(0.9)-\left(1-0.9^{2}\right) \mathcal{K}(0.9)\right]}{0.9 \pi}-\frac{\omega(1)}{p_{0}\left(p_{0}+1\right) 2^{1 / p_{0}} \eta(0.9)^{1-1 / p_{0}}} \\
& =0.522306 \cdots-0.46787 \cdots=0.054436 \cdots>0
\end{aligned}
$$

for $0.9 \leq r<1$.

Therefore, it follows from the monotonicity of $\phi_{p_{0}}^{\prime}(r)$ on $(9 / 10,1)$ that $\phi_{p_{0}}(r)<\phi_{p_{0}}(1)=$ $2\left[2 / \pi-1 /\left(1+p_{0}\right)^{1 / p_{0}}\right]=0$ for $0<r<1$.

Case $35 / 2<p<p_{0}$.

Taking the Taylor series of $\phi_{p}(r)$ at $r=0$ yields

$$
\phi_{p}(r)=\left(\frac{5}{12}-\frac{p}{6}\right) r^{2}+\frac{\left(149-144 p+24 p^{2}+16 p^{3}\right) r^{4}}{2,880}+o\left(r^{4}\right)
$$

From (2.24) we clearly see that there exists a sufficiently small $\delta_{1}>0$ such that $\phi_{p}(r)<0$ for $r \in\left(0, \delta_{1}\right)$ if $p>5 / 2$. If $p<p_{0}$, then $\phi_{p}(1)=2\left[2 / \pi-1 /(1+p)^{1 / p}\right]>0$. By the continuity of $\phi_{p}(r)$ with respect to $r$, there exists a sufficiently small $\delta_{2}>0$ such that $\phi_{p}(r)>0$ for $r \in\left(\delta_{2}, 1\right)$. 


\section{Main results}

Theorem 3.1 Inequality $L_{-1}(a, b)<\operatorname{AG}(a, b)<L_{-1 / 2}(a, b)$ holds for all $a, b>0$ with $a \neq b$, where $L_{-1}(a, b)$ and $L_{-1 / 2}(a, b)$ are the best possible lower and upper generalized logarithmic mean bounds for the arithmetic-geometric mean $\mathrm{AG}(a, b)$, respectively.

Proof Firstly, from (1.5) we clearly see that $L_{-1}(a, b)<\operatorname{AG}(a, b)$ for all $a, b>0$ with $a \neq b$.

Next, we prove that $\operatorname{AG}(a, b)<L_{-1 / 2}(a, b)$ for all $a, b>0$ with $a \neq b$. Since $\operatorname{AG}(a, b)$ and $L_{p}(a, b)$ are symmetric and homogeneous of degree 1 , without loss of generality, it suffices to give an assumption that $a=1>b$. Let $t=b \in(0,1), r=(1-t) /(1+t)$, then (1.1) and (1.3) lead to

$$
\begin{aligned}
\operatorname{AG}(a, b)-L_{-1 / 2}(a, b) & =\frac{\pi}{2 \mathcal{K}\left(\sqrt{1-t^{2}}\right)}-\left[\frac{1-t}{2(1-\sqrt{t})}\right]^{2} \\
& =\frac{1}{1+r}\left[\frac{\pi}{2 \mathcal{K}}-\left(\frac{r}{\sqrt{1+r}-\sqrt{1-r}}\right)^{2}\right] \\
& =\frac{h(r)}{2(1+r) \mathcal{K}(r)},
\end{aligned}
$$

where

$$
h(r)=\pi-\left(1+r^{\prime}\right) \mathcal{K}(r) .
$$

We can rewrite $h(r)$ as

$$
h(r)=\pi-\lambda\left(r^{\prime}\right) \cdot \frac{\mathcal{K}}{\log \left(e^{2} / r^{\prime} 2\right)},
$$

where $\lambda\left(r^{\prime}\right)=\left(1+r^{\prime}\right) \log \left(e^{2} / r^{\prime}\right)$.

A simple calculation yields

$$
\begin{aligned}
& \lambda^{\prime}\left(r^{\prime}\right)=1-\frac{1}{r^{\prime}}-\log r^{\prime}, \\
& \lambda^{\prime}(1)=0, \\
& \lambda^{\prime \prime}\left(r^{\prime}\right)=\frac{1-r^{\prime}}{r^{\prime}}>0 .
\end{aligned}
$$

Equations (3.3)-(3.5) lead to the conclusion that $\lambda\left(r^{\prime}\right)$ is strictly decreasing on $(0,1)$ with respect to $r^{\prime}$. Moreover, the function $r^{\prime}=\sqrt{1-r^{2}}$ is strictly decreasing on $(0,1)$. Hence the function $\lambda\left(r^{\prime}\right)$ is strictly increasing on $(0,1)$ with respect to $r$. It follows from (3.2) and Lemma 2.2(3) that $h(r)$ is strictly decreasing on $(0,1)$. This implies that $h(r)<0$ for $0<r<1$ together with $h(0)=0$.

Therefore, $\operatorname{AG}(a, b)<L_{-1 / 2}(a, b)$ for all $a, b>0$ with $a \neq b$ follows from (3.1) and $h(r)<0$. Finally, we prove that $L_{-1}(a, b)$ and $L_{-1 / 2}(a, b)$ are the best possible lower and upper generalized logarithmic mean bounds for the arithmetic-geometric mean $\operatorname{AG}(a, b)$.

For any $0<\varepsilon<1 / 2$ and $0<x<1$, it follows from (1.1) and (1.3) that

$$
\begin{aligned}
\lim _{x \rightarrow 0}\left[\operatorname{AG}(1, x)-L_{-1+\varepsilon}(1, x)\right] & =\lim _{x \rightarrow 0}\left\{\frac{\pi}{2 \mathcal{K}\left(\sqrt{1-x^{2}}\right)}-\left[\frac{1-x^{\varepsilon}}{\varepsilon(1-x)}\right]^{\frac{1}{\varepsilon-1}}\right\} \\
& =-\varepsilon^{\frac{1}{1-\varepsilon}}
\end{aligned}
$$


and making use of the Taylor expansion as $x \rightarrow 0$, one has

$$
\begin{aligned}
& \operatorname{AG}(1,1-x)-L_{-1 / 2-\varepsilon}(1,1-x) \\
& \quad=\frac{\pi}{2 \mathcal{K}\left(\sqrt{2 x-x^{2}}\right)}-\left[\frac{1-(1-x)^{1 / 2-\varepsilon}}{(1 / 2-\varepsilon) x}\right]^{-\frac{2}{1+2 \varepsilon}} \\
& \quad=\left[1-\frac{x}{2}-\frac{x^{2}}{16}+o\left(x^{2}\right)\right]-\left[1-\frac{x}{2}-\frac{3+2 \varepsilon}{48} x^{2}+o\left(x^{2}\right)\right] \\
& \quad=\frac{\varepsilon}{24} x^{2}+o\left(x^{2}\right) .
\end{aligned}
$$

Equations (3.6) and (3.7) imply that for any $0<\varepsilon<1 / 2$ there exist $\delta_{1}=\delta_{1}(\varepsilon) \in(0,1)$ and $\delta_{2}=\delta_{2}(\varepsilon) \in(0,1)$ such that $\operatorname{AG}(1, x)<L_{-1+\varepsilon}(1, x)$ for $x \in\left(0, \delta_{1}\right)$ and $\operatorname{AG}(1,1-x)>$ $L_{-1 / 2-\varepsilon}(1,1-x)$ for $x \in\left(0, \delta_{2}\right)$.

Theorem 3.2 Inequality $L_{5 / 2}(a, b)<T(a, b)<L_{p_{0}}(a, b)$ holds for all $a, b>0$ with $a \neq b$, where $p_{0}$ is defined as in Lemma 2.3 and $L_{5 / 2}(a, b), L_{p_{0}}(a, b)$ are the best possible lower and upper generalized logarithmic mean bounds for the Toader mean $T(a, b)$, respectively.

Proof From (1.1) and (1.4) we clearly see that both $T(a, b)$ and $L_{p}(a, b)$ are symmetric and homogeneous of degree 1 . Without loss of generality, we assume that $a=1>b$. Let $t=b \in$ $(0,1), r=(1-t) /(1+t)$, then from (1.1) and (1.4) together with (2.2) we have

$$
\begin{aligned}
T(a, b)-L_{p}(a, b) & =\frac{2}{\pi} \mathcal{E}\left(\sqrt{1-t^{2}}\right)-\left[\frac{1-t^{p+1}}{(p+1)(1-t)}\right]^{1 / p} \\
& =\frac{2}{\pi} \mathcal{E}\left(\frac{2 \sqrt{r}}{1+r}\right)-\frac{1}{1+r}\left[\frac{(1+r)^{p+1}-(1-r)^{p+1}}{2(p+1) r}\right]^{1 / p} \\
& =\frac{1}{1+r}\left[\frac{2}{\pi}\left(2 \mathcal{E}-r^{\prime 2} \mathcal{K}\right)-\left(\frac{(1+r)^{p+1}-(1-r)^{p+1}}{2(p+1) r}\right)^{1 / p}\right] \\
& =\frac{\phi_{p}(r)}{(1+r)}
\end{aligned}
$$

where $\phi_{p}(r)$ is defined as in Lemma 2.8 .

Therefore, Theorem 3.2 follows from (3.8) and Lemma 2.8.

\section{Corollaries and remarks}

From Theorem 3.1 we get a lower bound for the complete elliptic integral of the first kind $\mathcal{K}(r)$ as follows.

\section{Corollary 4.1 Inequality}

$$
\mathcal{K}(r)>\frac{2 \pi\left[1+\sqrt{1-r^{2}}-2\left(1-r^{2}\right)^{1 / 4}\right]}{\left(1-\sqrt{1-r^{2}}\right)^{2}}
$$

holds for all $r \in(0,1)$.

Remark 4.1 We define $H(r)=2 \pi\left[1+\sqrt{1-r^{2}}-2\left(1-r^{2}\right)^{1 / 4}\right] /\left(1-\sqrt{1-r^{2}}\right)^{2}$. Computational and numerical experiments show that the lower bound in (4.1) can be regarded as an approximation of $\mathcal{K}(r)$ for some $r \in(0,1)$, refer to Table 1 for numerical values. 
Table 1 Comparison of $\mathcal{K}(r)$ with $H(r)$ for some $r \in(0,1)$

\begin{tabular}{lll}
\hline $\boldsymbol{r}$ & $\mathcal{K}(\boldsymbol{r})$ & $\boldsymbol{H}(\boldsymbol{r})$ \\
\hline 0.1 & $1.57474555615 \cdots$ & $1.5747455614 \cdots$ \\
0.2 & $1.5868678474 \cdots$ & $1.5868678471 \cdots$ \\
0.3 & $1.608048619 \cdots$ & $1.608048612 \cdots$ \\
0.4 & $1.63999986 \cdots$ & $1.63999977 \cdots$ \\
0.5 & $1.68575035 \cdots$ & $1.68574965 \cdots$ \\
0.6 & $1.75075380 \cdots$ & $1.75074958 \cdots$ \\
0.7 & $1.84569400 \cdots$ & $1.84567106 \cdots$ \\
0.8 & $1.99530278 \cdots$ & $1.99517293 \cdots$ \\
\hline
\end{tabular}

Theorem 3.2 enables us to give new bounds for the complete elliptic integrals of the second kind $\mathcal{E}(r)$.

\section{Corollary 4.2 Inequality}

$$
\frac{\pi}{2}\left[\frac{2\left(1-\left(1-r^{2}\right)^{7 / 4}\right)}{7\left(1-\sqrt{1-r^{2}}\right)}\right]^{2 / 5}<\mathcal{E}(r)<\frac{\pi}{2}\left[\frac{1-\left(1-r^{2}\right)^{\left(p_{0}+1\right) / 2}}{\left(p_{0}+1\right)\left(1-\sqrt{1-r^{2}}\right)}\right]^{1 / p_{0}}
$$

holds for all $r \in(0,1)$, where $p_{0}=3.15295 \cdots$ is defined as in Lemma 2.3 .

\section{Competing interests}

The authors declare that they have no competing interests.

\section{Authors' contributions}

All authors contributed equally to the writing of this paper. All authors read and approved the final manuscript.

\section{Author details}

${ }^{1}$ College of Mathematics and Statistics, Hunan University of Finance and Economics, Changsha, 410205, China.

2Department of Mathematics, Hangzhou Normal University, Hangzhou, 311121, China.

\section{Acknowledgements}

The authors wish to thank the anonymous referees for their careful reading of the manuscript and their fruitful comments and suggestions. This research was supported by the National Natural Science Foundation of China (Grant no. 11301127), the general project Foundation of the Department of Education of Hunan Province (Grant no. 16C0265) and the scientific research Foundation for Young teachers in Hunan University of Finance and Economics (Grant no. Q201501).

\section{Publisher's Note}

Springer Nature remains neutral with regard to jurisdictional claims in published maps and institutional affiliations.

Received: 15 February 2017 Accepted: 13 April 2017 Published online: 05 May 2017

\section{References}

1. Stolarsky, KB: Generalizations of the logarithmic mean. Math. Mag. 48, 87-92 (1975)

2. Chu, YM, Hou, SW, Gong, WM: Inequalities between logarithmic, harmonic, arithmetic and centroidal means. J. Math. Anal. 2(2), 1-5 (2011)

3. Chu, YM, Wang, MK: Optimal inequalities between harmonic, geometric, logarithmic, and arithmetic-geometric means. J. Appl. Math. 2011, Article ID 618929 (2011)

4. Qiu, YF, Wang, MK, Chu, YM, Wang, GD: Two sharp inequalities for Lehmer mean, identric mean and logarithmic mean. J. Math. Inequal. 5(3), 301-306 (2011)

5. Chu, YM, Xia, WF: Two optimal double inequalities between power mean and logarithmic mean. Comput. Math. Appl. 60(1), 83-89 (2010)

6. Xia, WF, Chu, YM, Wang, GD: The optimal upper and lower power mean bounds for a convex combination of the arithmetic and logarithmic means. Abstr. Appl. Anal. 2010, Article ID 604804 (2010)

7. Xia, WF, Chu, YM: Optimal inequalities related to the logarithmic, identric, arithmetic and harmonic means. Rev. Anal. Numér. Théor. Approx. 39(2), 176-183 (2010)

8. Chu, YM, Xia, WF: Inequalities for generalized logarithmic means. J. Inequal. Appl. 2009, Article ID 763252 (2009)

9. Shi, HN, Wu, SH: Refinement of an inequality for the generalized logarithmic mean. Chin. Q. J. Math. 23(4), 594-599 (2008)

10. Qi, F, Chen, SX, Chen, CP: Monotonicity of ratio between the generalized logarithmic means. Math. Inequal. Appl. 10(3), 559-564 (2007) 
11. Li, X, Chen, CP, Qi, F: Monotonicity result for generalized logarithmic means. Tamkang J. Math. 38(2), 177-181 (2007)

12. Chen, CP, Qi, F: Monotonicity properties for generalized logarithmic means. Aust. J. Math. Anal. Appl. 1(2), Article 2 (2004)

13. Mond, B, Pearce, CEM, Pečarić, J: The logarithmic mean is a mean. Math. Commun. 2(1), 35-39 (1997)

14. Kahlig, P, Matkowski, J: Functional equations involving the logarithmic mean. Z. Angew. Math. Mech. 76(7), 385-390 (1996)

15. Pittenger, AO: The logarithmic mean in $n$ variables. Am. Math. Mon. 92(2), 99-104 (1985)

16. Stolarsky, KB: The power and generalized logarithmic means. Am. Math. Mon. 87(7), 545-548 (1980)

17. Lin, TP: The power mean and the logarithmic mean. Am. Math. Mon. 81, 879-883 (1974)

18. Anderson, GD, Vamanamurthy, MK, Vuorinen, MK: Conformal Invariants, Inequalities, and Quasiconformal Maps. Wiley, New York (1997)

19. Toader, G: Some mean values related to the arithmetic-geometric mean. J. Math. Anal. Appl. 218, 358-368 (1998)

20. Carlson, BC, Vuorinen, MK: Inequality of the AGM and the logarithmic mean. SIAM Rev. 33, 653-654 (1991)

21. Sándor, J: On certain inequalities for means. J. Math. Anal. Appl. 189, 602-606 (1995)

22. Neuman, E, Sándor, J: On certain means of two arguments and their extensions. Int. J. Math. Math. Sci. 16, 981-993 (2003)

23. Yang, ZH: A new proof of inequalities for Gauss compound mean. Int. J. Math. Anal. 4, 1013-1018 (2010)

24. Vamanamurthy, MK, Vuorinen, MK: Inequalities for means. J. Math. Anal. Appl. 183, 155-166 (1994)

25. Borwein, JM, Borwein, PB: Inequalities for compound mean iterations with logarithmic asymptotes. J. Math. Anal. Appl. 177, 572-582 (1993)

26. Vuorinen, MK: Hypergeometric functions in geometric function theory. In: Special Functions and Differential Equations, pp. 119-126. Allied Publ., New Delhi (1998)

27. Qiu, SL, Shen, JM: On two problems concerning means. J. Hangzhou Inst. Electron. Eng. 17, 1-7 (1997) (in Chinese)

28. Barnard, RW, Pearce, K, Richards, KC: An inequality involving the generalized hypergeometric function and the arc length of an ellipse. SIAM J. Math. Anal. 31, 693-699 (2000)

29. Alzer, H, Qiu, SL: Monotonicity theorems and inequalities for the complete elliptic integrals. J. Comput. Appl. Math. $172,289-312$ (2004)

30. Chu, YM, Wang, MK: Optimal Lehmer mean bounds for the Toader mean. Results Math. 61, 223-229 (2012)

31. Guo, BN, Qi, F: Some bounds for the complete elliptic integrals of the first and second kinds. Math. Inequal. Appl. 14, 323-334 (2011)

32. Chu, YM, Wang, MK, Qiu, SL, Qiu, YF: Sharp generalized Seiffert mean bounds for Toader mean. Abstr. Appl. Anal. 2011, Article ID 605259 (2011)

33. Chu, YM, Wang, MK: Inequalities between arithmetic-geometric, Gini, and Toader means. Abstr. Appl. Anal. 2012, Article ID 830585 (2012)

\section{Submit your manuscript to a SpringerOpen ${ }^{\circ}$ journal and benefit from:}

- Convenient online submission

Rigorous peer review

- Immediate publication on acceptance

- Open access: articles freely available online

- High visibility within the field

- Retaining the copyright to your article 